\title{
IUFOST2006/1371 Evaluation of the Physiological Effects of Conjugated Alpha-Linolenic Acids (CLnA)
}

\author{
M. Plourde ${ }^{\mathrm{a}}$, P. Angers ${ }^{\mathrm{b}}$ and J.-L. Sébédio ${ }^{\mathrm{c}}$ \\ ${ }^{a}$ Université de Sherbrooke, centre de recherche sur le vieillissement, 1036 Belvédère sud, J1H4C4 \\ Sherbrooke, Canada \\ buniversité Laval, Faculté des Sciences de l'Agriculture et de l'Alimentation, Département d'alimentation et \\ de nutrition, G1K 7P4 Québec, Canada \\ ' INRA-Université d'Auvergne, Laboratoire de Nutrition Humaine, 58 rue de Montalembert,, 63009 \\ Clermont-Ferrand, France \\ melanie.plourde.1@ulaval.ca
}

Conjugated alpha-linolenic acids (CLnA) are found in milk fat. The physiological effects of feeding a mixture of CLnA composed 18:3 of cis-9,trans-11,cis-15 and cis-9,trans-13,cis-15 at equimolar concentration was evaluated in vivo. We have first demonstrated that the CLnA mixture was absorbed, incorporated and metabolized when given to rats in physiological conditions. The metabolites formed were incorporated into plasma and liver phospholipids. However, only one CLnA isomer, the 18:3 of cis-9,trans-11,cis-15, have been elongated and desaturated into conjugated isomers of eicosapentaenoic acid (EPA) and docosahexaenoic acid (DHA). We hypothesized that this isomer could have a greater physiological effect due to its elongation/desaturation pathway metabolism. In another study, we investigated the effect of CLnA mixture on cardiovascular disease and found that it increased small dense LDL in hamsters fed a hypercholesterolemic diet with CLnA mixture. However, this increase had no repercussions on the formation of fatty streaks in the aortas. Another experiment has been done on the influence of feeding CLnA mixture on body fat mass and feed efficiency on male and female mice. A tendency to decrease the body fat mass was observed in female and no liver fat accumulation was observed in the animals. Finally, in rats induced chemically mammary tumors and fed the CLnA mixture, a significant decrease of tumors incidence was observed compared to control group. However, no impact of the diet was observed for the growth of tumors with this diet and compared to control group. In conclusion, the CLnA mixture used in this study show slight physiological effects. However, it would be interesting to investigate single CLnA isomers physiological effects to see if there is some differences between both isomers used in this study. 